OPEN ACCESS

Edited by:

Michael Strupp

Ludwig Maximilian University of

Munich, Germany

Reviewed by:

Shinichi Iwasaki,

The University of Tokyo, Japan Derek Alexander Hamilton, University of New Mexico,

United States

Klaus Jahn,

Schön Klinik, Germany

*Correspondence:

Bieke Dobbels

bieke.dobbels@uza.be

Specialty section: This article was submitted to Neuro-Otology, a section of the journal

Frontiers in Neurology

Received: 06 April 2020

Accepted: 10 June 2020

Published: 11 August 2020

Citation:

Dobbels B, Mertens G, Gilles A, Moyaert J, van de Berg R, Fransen E, Van de Heyning $P$ and Van Rompaey $V$ (2020) The Virtual Morris Water Task in

64 Patients With Bilateral

Vestibulopathy and the Impact of Hearing Status. Front. Neurol. 11:710.

doi: 10.3389/fneur.2020.00710

\section{The Virtual Morris Water Task in 64 Patients With Bilateral Vestibulopathy and the Impact of Hearing Status}

\author{
Bieke Dobbels ${ }^{1,2,3 *}$, Griet Mertens ${ }^{1,2}$, Annick Gilles ${ }^{1,2}$, Julie Moyaert ${ }^{2}$, \\ Raymond van de Berg ${ }^{4,5}$, Erik Fransen ${ }^{6}$, Paul Van de Heyning ${ }^{1,2}$ and \\ Vincent Van Rompaey ${ }^{1,2}$
}

${ }^{1}$ Faculty of Medicine and Health Sciences, University of Antwerp, Antwerp, Belgium, ${ }^{2}$ Department of Otorhinolaryngology and Head and Neck Surgery, Antwerp University Hospital, Edegem, Belgium, ${ }^{3}$ Department of Otorhinolaryngology and Head and Neck Surgery, Zuyderland Medical Center, Heerlen, Netherlands, ${ }^{4}$ Division of Balance Disorders, Department of Otorhinolaryngology and Head and Neck Surgery, Maastricht University Medical Center, Maastricht, Netherlands, ${ }^{5}$ Faculty of Physics, Tomsk State University, Tomsk, Russia, ${ }^{6}$ StatUa Center for Statistics, University of Antwerp, Antwerp, Belgium

Background: Previous studies have demonstrated spatial cognitive deficits in patients with bilateral vestibulopathy (BVP). However, BVP patients frequently present with a concomitant sensorineural hearing loss, which is a well-established risk factor of cognitive impairment and incident dementia. Nonetheless, previous research on spatial cognitive deficits in BVP patients have not taken hearing status into account.

Objective: This study aims to compare spatial cognition of BVP patients with healthy controls, with analyses adjusting for hearing status.

Methods: Spatial cognition was assessed in 64 BVP patients and 46 healthy controls $(\mathrm{HC})$ by use of the Virtual Morris Water Task (VMWT). All statistical analyses were adjusted for hearing (dys)function, sex, age, education, and computer use.

Results: Overall, patients with BVP performed worse on all outcome measures of the VMWT. However, these differences between BVP patients and healthy controls were not statistically significant. Nonetheless, a statistically significant link between sensorineural hearing loss and spatial cognition was observed. The worse the hearing, the longer subjects took to reach the hidden platform in the VMWT. Furthermore, the worse the hearing, the less time was spent by the subjects in the correct platform quadrant during the probe trial of the VMWT.

Conclusion: In this study, no difference was found regarding spatial cognition between BVP patients and healthy controls. However, a statistically significant link was observed between sensorineural hearing loss and spatial cognition.

Keywords: spatial cognition, vestibular loss, hearing loss, hippocampus, Morris Water Maze

\section{INTRODUCTION}

A growing body of literature recognizes that the function of the vestibular system goes far beyond balance and gaze stability. Both animal and human research suggests that the vestibular system plays a critical role in cognition (1-4). According to the Diagnostic and Statistical Manual of Mental Disorders (DSM-V), cognitive functioning can be subdivided into six domains: language, learning 
and memory, social cognition, attention, executive function, and visuospatial abilities (5). Of these, it seems that visuospatial abilities, which compromises spatial memory and navigation, is by far the most studied cognitive domain in animals and humans with loss of peripheral vestibular input $(2,4,6)$. For example, spatial cognition has been studied in patients with vestibular loss using the Virtual Morris Water Task (VMWT) $(3,7,8)$. This is a virtual version of the Morris Water Maze, considered the golden standard for assessing spatial cognition in rodents (9). Impaired spatial cognition has repeatedly been observed in patients with bilateral vestibulopathy $(B V P)(3,7)$. Patients with BVP suffer from a bilateral partial or complete loss of function of the vestibular structures of the inner ear, vestibular nerves, or a combination of both. BVP patients often present with oscillopsia and gait imbalance as primary complaints (10).

The link between spatial cognition and the vestibular system is of clinical importance for several reasons. First, cognitive training might yield therapeutic opportunities for BVP. Conventional treatment for patients with BVP is limited to counseling and intensive daily vestibular physical therapy to improve gaze and postural stabilization (11). However, these therapeutic strategies often remain insufficient (12). Although the utility of cognitive training has been demonstrated to enhance balance in the elderly and in patients with mild cognitive impairment and dementia, cognitive training is not included in the current treatment of BVP $(13,14)$. According to a recent computational model, cognitive training facilitates the central compensation process in BVP patients by increasing the knowledge about self-motion (15).

Second, interest has been directed toward the link between cognitive impairment and the vestibular system because of the rising prevalence of dementia. As in BVP patients, impaired spatial cognition is among the most frequently observed cognitive deficits in patients with dementia. One of the hallmark symptoms of Alzheimer's disease is wandering behavior and loss of topographic memory (16). The vestibular system, more than any other sensory system, makes widespread cortical projections, including to the hippocampus. The hippocampus is thought to play a key role in the neuronal substrate underlying spatial cognitive deficits in BVP patients (4). For instance, in a leading study by Brandt et al., BVP patients showed bilateral hippocampal atrophy and spatial cognitive deficits (7). Interestingly, in Alzheimer's disease, damage to the hippocampus is the most important anatomopathological feature (17).

Furthermore, several studies have found significantly poorer vestibular function in patients with dementia compared with their healthy peers (18-20).

These observations have led to the hypothesis that vestibular loss might cause cognitive decline and thus may contribute to the development of dementia. Given the rising prevalence of dementia and the lack of curative treatment, the identification of potentially modifiable risk factors is crucial (21).

In the previous literature, however, little attention has been paid to the hearing status of vestibular patients when drawing conclusions about the link between cognitive decline and the vestibular system. A systematic review pointed out that none of the studies investigating cognition in BVP patients have adjusted their analysis for the hearing status of the enrolled subjects (6).
However, because of the close anatomical relationship between the vestibular system and the cochlea, hearing loss is observed in up to half of BVP patients $(22,23)$. Hearing loss is a wellestablished risk factor for dementia (24-26). Therefore, it is uncertain whether the cognitive deficits observed in BVP patients can be solely attributed to their vestibular loss as previously assumed. The frequently associated hearing loss in BVP patients might also play an essential role in their cognitive impairment (6).

The goal of this study is to compare spatial cognitive performance, assessed using the VMWT, of BVP patients with healthy controls. In contrast to previous studies, the analyses in this study were specifically designed to take the hearing loss of BVP patients into account.

\section{METHODS}

\section{Study Design}

The current study was a single-center, prospective, crosssectional study, recruiting from October 2017 until August 2018 at the Antwerp University Hospital. The study was approved by the local ethics committee of the Antwerp University Hospital/University of Antwerp (protocol number 16/42/426) and informed consent was obtained in all study participants before the start of the study. The study was registered on ClinicalTrials.gov (NCT03690817). The majority of the enrolled participants received general cognitive assessment at another scheduled appointment on a different day. Results have been published earlier (27).

\section{Study Participants}

BVP patients were recruited from the Otorhinolaryngology, Head and Neck Surgery Department at Antwerp University Hospital, Belgium. Inclusion criteria for the BVP group were (1) BVP disease duration of more than 6 months and (2) definite diagnosis of BVP as defined by the diagnostic criteria of the Bárány Society (28):

a) Horizontal angular vestibulo-ocular reflex (VOR) gain $<0.6$ measured by the video head impulse test (vHIT), and/or

b) Reduced caloric response (sum of bithermal, 30 and $44^{\circ}$, maximum peak slow phase velocity (SPV) on each side $\left.<6^{\circ} / \mathrm{s}\right)$, and/or

c) Reduced horizontal angular VOR gain $<0.1$ upon sinusoidal stimulation on a rotatory chair.

Control participants were recruited by means of the population registries at the local city councils in southern Antwerp (Belgium), by advertisements in the hospital, and by approaching friends, family, and colleagues. Only control subjects with no history of vertigo, scores $<5$ on the Dizziness Handicap Inventory, and normal hearing thresholds at $0.25-8 \mathrm{kHz}$, based on age and sex (defined by the BS 6951:1988, EN 27029:1991, and ISO 7029-1984 standards), were enrolled in the study.

The following additional inclusion criteria were applied for both BVP patients and healthy controls: (1) age $\geq 18$ years, (2) fluency in Dutch, (3) no history of neurological diseases (e.g., dementia, Parkinson's disease, cerebrovascular accident, etc.), (4) 
absence of clinical signs indicating dementia or mild cognitive impairment, and (5) normal or appropriate corrected vision.

Regarding the necessity of computer use in the VMWT, all participants were asked about their frequency of computer use (daily vs. 2-5 days/week vs. seldom/never). Education of all participants was categorized as primary school, lower secondary school, upper secondary school, and college/university.

\section{Vestibular Testing}

By enrollment in the study, all BVP patients received new neuro-otological testing on site. The evaluation of the lower and mid frequencies function of the lateral semi-circular canals was performed by electronystagmography with bithermal caloric tests and rotatory chair test (Nystagliner Toennies, Germany). At our clinic, rotatory chair tests are performed using sinusoidal rotation $(0.05 \mathrm{~Hz})$ with a peak velocity of $60^{\circ} / \mathrm{s}$ (29). More detailed methodology and normative data were previously described (29). High-frequency function of all six semi-circular canals was measured by the vHIT. In the standard procedure used at our clinic, 10 valid head impulses are required for each canal. Angular head velocity was determined by three mini-gyroscopes, eye velocity by means of an infrared camera recording the right eye, all incorporated in commercially available vHIT goggles (Otometrics, Taastrup, Denmark). VOR gain was defined as the ratio of the area under the eye velocity curve to the head velocity curve from the impulse onset until the head velocity was again 0 (30).

\section{The Virtual Morris Water Task (VMWT)}

To assess spatial learning and spatial memory retrieval, the VMWT was used. This task was designed by Derek Hamilton and was inspired by the original animal research tool, which is considered the gold standard for testing spatial cognition in rodents $(9,31)$. A 15.6-in. PC laptop monitor was used to display the virtual environment generated by the VMWT software version 1.10 (Neuro Investigations). In this task, participants had to navigate toward a hidden platform as fast as possible. The virtual environment consisted of a round pool, located in the middle of a square room. Each wall of the room contained a different visual cue on which a participant could rely to find his way to the hidden platform. The cues were positioned in such a way that the platform could not be encountered by simply moving toward a single cue (see Figure 1). On the computer screen, a first-person view of the virtual environment was shown. Participants could move in the pool by using the arrow keys on the keyboard. Backward movement or up-down movement was not possible.

Before testing, all participants were given the same written instructions. Afterwards, time to ask questions to the examiner was foreseen. In both groups, four phase trials were performed:

\section{Phase I-Exploration Trial}

The first part consisted of one block of four trials with a hidden platform. Participants were familiarized with the concept of the game and the use of the key arrows. By observing the participant, the examiner checked for good understanding of the task. When necessary, supplemental explanations were given.

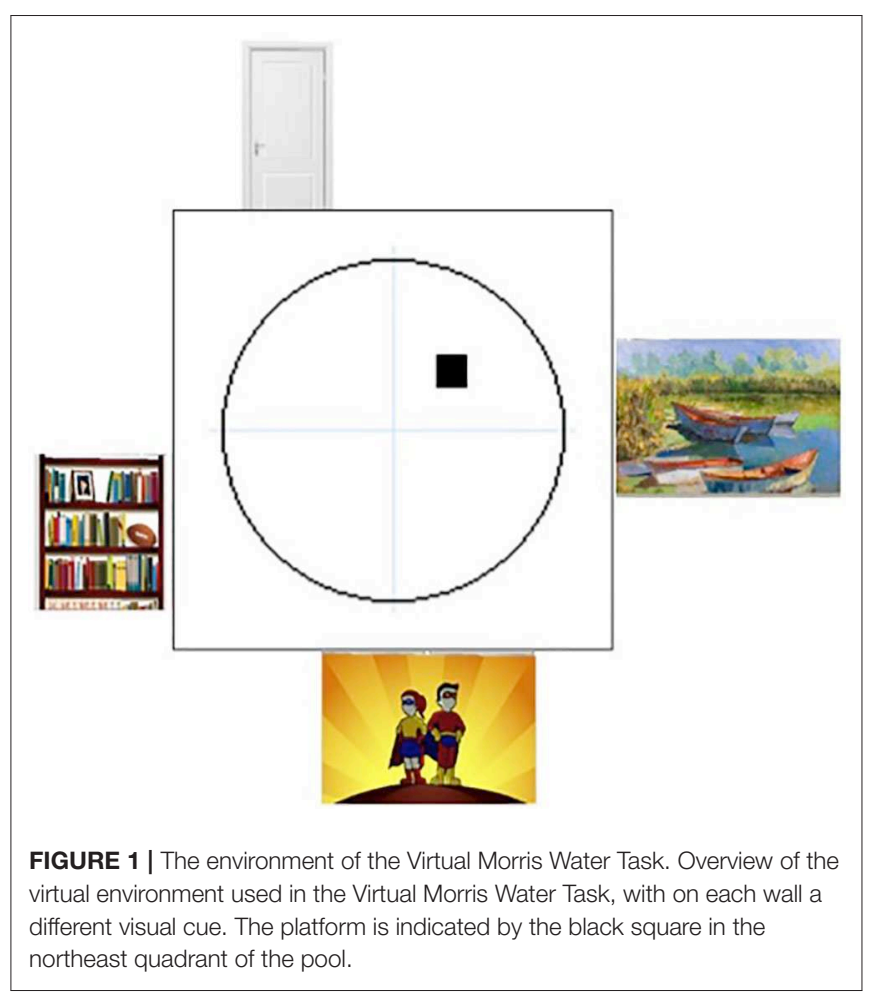

\section{Phase II-Hidden Trial}

The test was started with 20 hidden platform trials. A virtual environment with different visual cues was used than in the exploration trial (see Figure 2). The hidden platform was located, in all trials, at the same spot in the northeastern quadrant of the pool. As it was submerged underneath the pool's surface, it was not visible to the participants. Starting locations during each trial were sampled pseudo-randomly from the four cardinal direction points of the pool. If participants were unable to find the hidden platform after $60 \mathrm{~s}$, the platform was made visible and a message appeared prompting the participant to swim to the platform. During each of these trials, three measures were computed:

- The latency, i.e., time to reach the platform.

- The covered path length, i.e., total distance traveled, divided by the pool diameter.

- The heading error, when the participant has traveled a distance $>25 \%$ of the pool diameter from the start position; the angular deviation is computed between the straight trajectory to the center of the platform and the starting position (see Figure 3).

Performance during these hidden platform trials represent a measure for spatial learning performance.

\section{Phase III-Probe Trial}

Subsequently, the platform was removed from the pool, unbeknownst to the participants. In this one trial, we measured the percentage of time a participant spent in the platform quadrant. A higher percentage was considered to be related to a better spatial memory retrieval of the participant. 


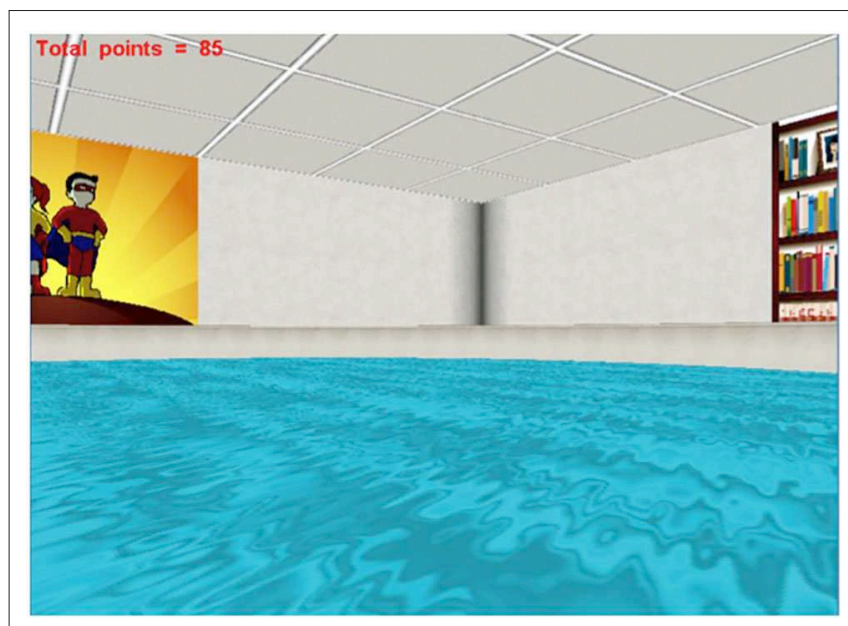

FIGURE 2 | The user's view during the Virtual Morris Water Task. Spatial cognition was assessed by the Virtual Morris Water Task. This figure shows the first-person view of the virtual environment, presented on a computer screen.

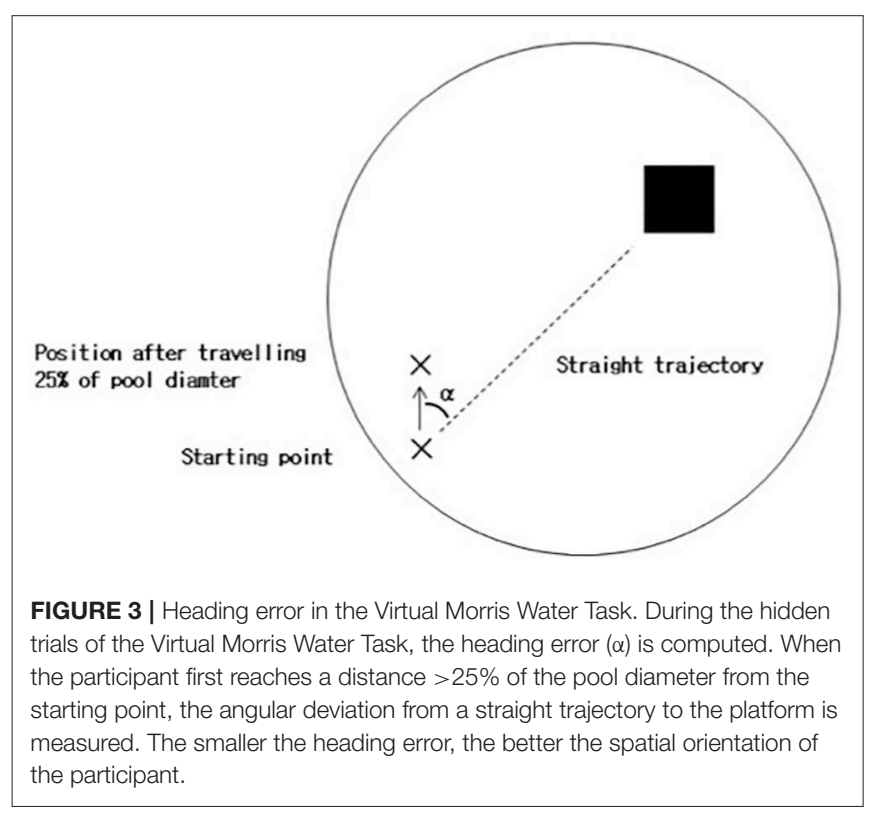

\section{Phase IV-Visible Trials}

The last part of the test represents a control task for motor condition. Participants had to perform eight visible trials in which the platform was visible and participants had to swim to the platform as soon as possible. Again, latency and path length were recorded for each trial.

\section{Hearing Assessment}

To correct cognitive outcome measures for the hearing status of the enrolled participants, a pure tone audiometry was performed. The unaided hearing thresholds were measured in a soundisolated booth. For air conduction, hearing thresholds were determined at $125,250,500,1,000,2,000,3,000,4,000,6,000$, and $8,000 \mathrm{~Hz}$ using a two-channel Interacoustics AC-40 audiometer and insert earphones. Bone conduction thresholds were tested at 250, 500, 1,000, 2,000, 3,000, and 4,000 Hz. The high Fletcher index, which is the mean of air conduction hearing thresholds at 1,2 , and $4 \mathrm{kHz}$, was calculated for both ears. The hearing status of a participant was defined by the high Fletcher index of the better-hearing ear.

\section{Data Collection and Statistical Analysis}

Data were stored in OpenClinica LLC (Waltham, MA, USA), a secured online database for electronic data registration and data management developed for clinical research. For statistical analyses, IBMS SPSS Statistics (IBM Corp. Released 2016. Version 24.0. Armonk, NY) and "R" was used (R: A language and environment for statistical computing. Released 2013. R Foundation for Statistical Computing, Vienna, Austria).

Depending on distribution, demographic data were analyzed with either $t$-test and $\chi^{2}$ test, or Mann-Whitney test and Fisher's exact test. Analogous to previous work, the 20 hidden platform trials were divided into three blocks: block 1 with trials $1-4$, block 2 with trials 5-12, and block 3 with trials 13-20. First, for each performance variable of the hidden trial (latency, path length, and heading error), a linear mixed model was fitted. A random effect of individual was added to account for the non-independence between observations from the same individual. Fixed effects included group (BVP vs. healthy controls), time (repeated measurements during the three blocks of trials), and their interaction. The latter interaction term evaluates whether there is a difference in learning between BVP patients and healthy controls during the VMWT. In other words, a statistically significant interaction term points out that during all 20 hidden trials, one group progressively found the platform faster compared with the other group, indicating a better learning curve in this group throughout the test.

In the absence of a significant interaction, linear mixed models were fitted for all performance variables of the hidden trials, with the main factors group (BVP vs. healthy controls) and time (repeated measurements during the three blocks of trials), without interaction term and hearing status as indicated by the high Fletcher index. Using these models, we evaluated whether there was a statistically significant different performance between the two participant's groups, BVP and healthy controls, across all trial blocks. Moreover, using this model, we assessed whether there was a statistically significant main effect of the hearing status on VMWT performance. In all of these models, the following covariates were added: age, sex, computer use, and education.

To compare the spatial memory retrieval during the probe trial, a multiple linear regression model was fitted with the main factors group (BVP vs. healthy controls) and hearing status (high Fletcher index of the better-hearing ear). Again, age, sex, computer use, and education were entered as covariates.

Finally, mean path lengths and latencies during the 10 visible trials were computed and used as dependent variables in a similar multiple linear regression model. 


\section{RESULTS}

\section{Participant Characteristics}

Sixty-four BVP patients with a mean age of $59 \pm 14$ years met the study inclusion criteria; $60 \%$ of them were male. Forty-six healthy controls with a mean age of $48 \pm 17$ were enrolled in the study; $44 \%$ of them were male (Table 1). The BVP group was gender matched to the control group. BVP patients were on average older, less educated, and had less computer experience

TABLE 1 | Demographic data.

\begin{tabular}{|c|c|c|c|}
\hline & $\begin{array}{l}\text { BVP patients } \\
\qquad n=64\end{array}$ & $\begin{array}{l}\text { Healthy controls } \\
\qquad n=46\end{array}$ & $P$-value \\
\hline Age (mean, SD) & $59(14)$ & $48(17)$ & $<0.05$ \\
\hline $\operatorname{Sex}(n, \%)$ & & & 0.1 \\
\hline Male & $38(60)$ & $20(32)$ & \\
\hline Female & $26(33)$ & $26(57)$ & \\
\hline Years of education (mean, SD) & $13(3)$ & $17(3)$ & $<0.05$ \\
\hline Computer use $(n, \%)$ & & & $<0.05$ \\
\hline Seldom/never & $15(27)$ & $2(6)$ & \\
\hline 2-5 days/week & $10(18)$ & $4(11)$ & \\
\hline Daily & $31(55)$ & $30(83)$ & \\
\hline \multicolumn{4}{|c|}{ Hearing performance: pure tone audiometry } \\
\hline $\begin{array}{l}\text { Fletcher index better-hearing } \\
\text { ear (mean, SD in } \mathrm{dB} \text { ) }\end{array}$ & $58(34)$ & $11(12)$ & $<0.05$ \\
\hline
\end{tabular}

BVP, bilateral vestibulopathy; $d B$, decibel. than healthy controls $(p<0.05)$. Hearing loss was more frequent in BVP patients (High Fletcher index $58 \pm 42 \mathrm{~dB}$ in BVP patients vs. $11 \pm 12 \mathrm{~dB}$ in healthy controls, $\mathrm{p}<0.05)$. To diagnose BVP, the Bárány Society criteria needed to be fulfilled (28). Forty percent of BVP patients met all Bárány Society criteria: a bilateral reduced response on caloric testing, rotatory chair test, and vHIT. In $30 \%$ of BVP patients, two out of three Bárány Society criteria were fulfilled, and in the remaining $30 \%$ of the BVP patients there was only found a vestibular hypofunction in one of the three vestibular tests (Figure 4). The mean gain of the left and right vHIT was, respectively, $4.2 \pm 0.3$ and $0.47 \pm 0.3$. The mean gain on the rotatory swing was $0.08 \pm 0.08$.

An underlying cause of vestibular loss could not be identified in $33.9 \%$ of BVP patients. With a prevalence of nearly $20 \%$, a mutation in the $\mathrm{COCH}$ gene causing DFNA9 was the most frequent underlying non-idiopathic etiology in our BVP cohort (35). In 16\% of BVP patients, an infectious cause was found (e.g., meningitis, neuritis, Lyme disease). Menière's disease and head trauma accounted for, respectively, 6 and 11\% of BVP causes. In four BVP patients, an ototoxic cause was suspected (three aminoglycosides antibiotics and one chemotherapy, not further specified).

\section{Results of the VMWT} Hidden Platform Trials: Spatial Learning

First, during the hidden platform trials, a significant main effect of time was found for all outcome measurements, indicating faster determination of the hidden platform location over time (see Table 2). No statistically significant interaction between

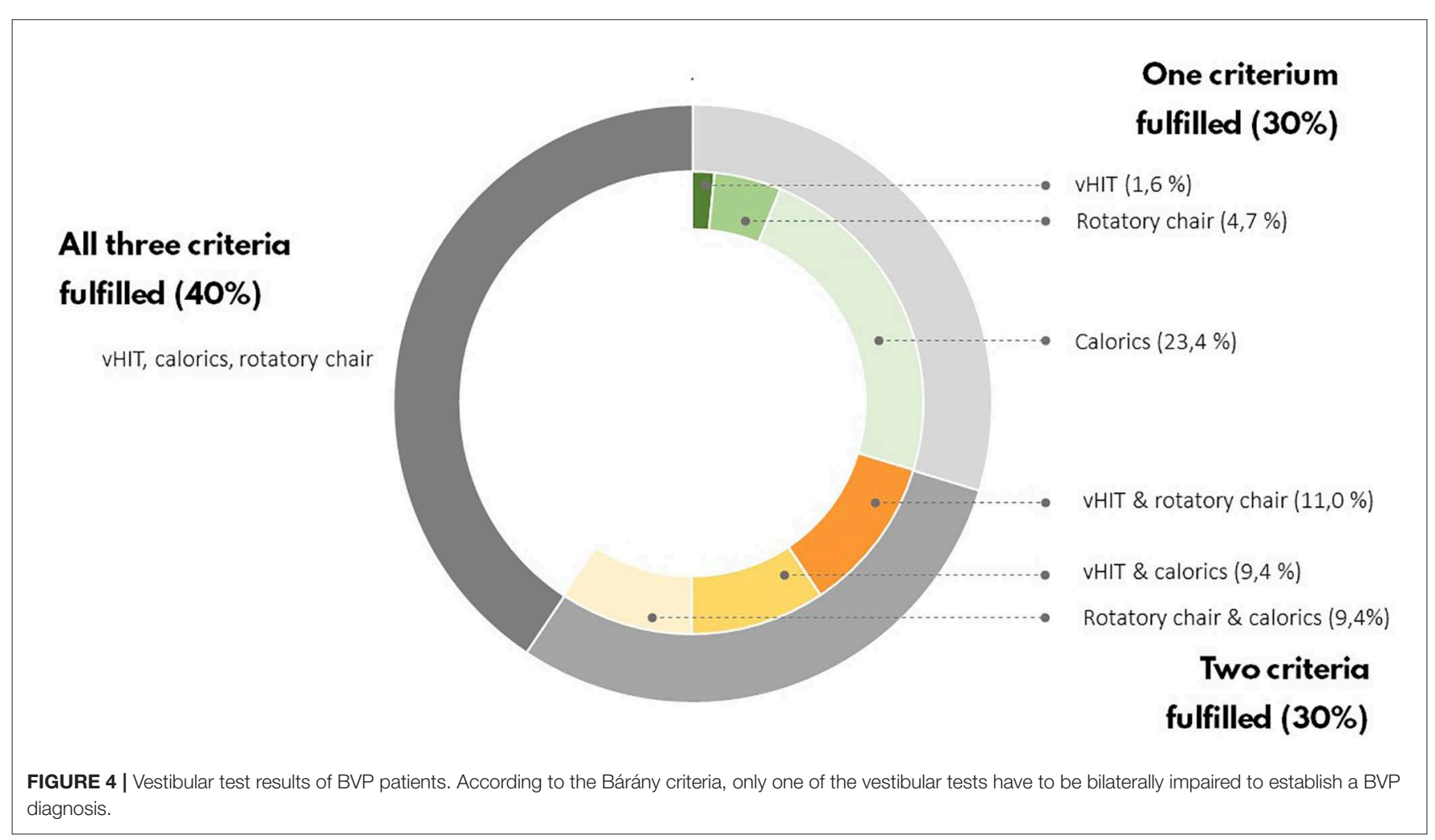


group $\times$ time was found in any of the outcome measures. This indicates that, regardless of the absolute outcome measurements in both groups, the learning curve in BVP patients was not significantly slower than in healthy controls. This is also illustrated by a similar slope of the curves in BVP patients and controls showed in Figures 5-7.

Second, as shown in Figures 5, 6, BVP patients took both more time and longer paths, compared with healthy controls, to reach the hidden platform during all three trial blocks $(1-4,5-$ 12, and 13-20). Likewise, the heading error of BVP patients was larger during all three trial blocks (see Figure 7). Importantly, in linear mixed models no significant group effect for latency, path length, or heading error was found. In other words, the worse

TABLE 2 | Results of the linear mixed models used for the hidden platform trials of the Virtual Morris Water Maze.

\begin{tabular}{lccc}
\hline $\begin{array}{l}\boldsymbol{P} \text {-value } \\
\text { (effect size if } \\
\mathbf{p}<\mathbf{0 . 0 5 )}\end{array}$ & $\begin{array}{c}\text { Group } \\
\text { (BVP vs. } \\
\text { healthy controls) }\end{array}$ & $\begin{array}{c}\text { Hearing status } \\
\text { (high Fletcher index of } \\
\text { better-hearing ear) }\end{array}$ & Age \\
\hline Latency & 0.16 & $0.006(0.11)$ & $<0.001(0.86)$ \\
Path length & 0.28 & 0.76 & $0.04(0.01)$ \\
Heading error & 0.32 & 0.56 & $<0.001(0.3)$
\end{tabular}

Outcome measures for spatial learning were (1) latency, (2) path length, and (3) heading error. All analyses adjusted for age, sex, computer use, education, and hearing loss. $A$ $p<0.05$ indicates a statistically significant main effect of the examined factor.

BVP, bilateral vestibulopathy. performance of BVP patients compared with healthy controls was not statistically significant. All these analyses were adjusted for hearing status, age, sex, computer use, and education. Third, a statistically significant association between hearing loss and spatial learning was seen. The higher the Fletcher index, the longer the latencies were during the hidden trials $(p=0.006$, effect size 0.11 ). As the Fletcher index increased by $1 \mathrm{~dB}$, the latency was 0.11 second longer. There was no significant effect of hearing loss on path length or heading error.

\section{Probe Trial: Spatial Memory Retrieval}

During the probe trial, BVP patients searched 38\% $( \pm 23.3)$ of their time in the correct quadrant, whereas healthy controls spent $52.1 \%( \pm 22.7)$ in the correct quadrant. However, this difference was not statistically significant between the two groups ( $p$-value in multiple linear regression model of groups $=0.9$ ).

Nonetheless, the analysis revealed a significant main effect of hearing loss on relative amount of time spent in the correct quadrant $(p=0.05, \beta-0.1)$. This indicates that the worse the hearing, the poorer the memory retrieval. The results of the probe trial are demonstrated in Figures 8, 9.

\section{Visible Trials: Motor Control Condition}

No significant effects of group or hearing status were found during the visible platform trials regarding latency, path length, and heading error. This indicates that BVP patients showed no difference in their motor control condition, compared with

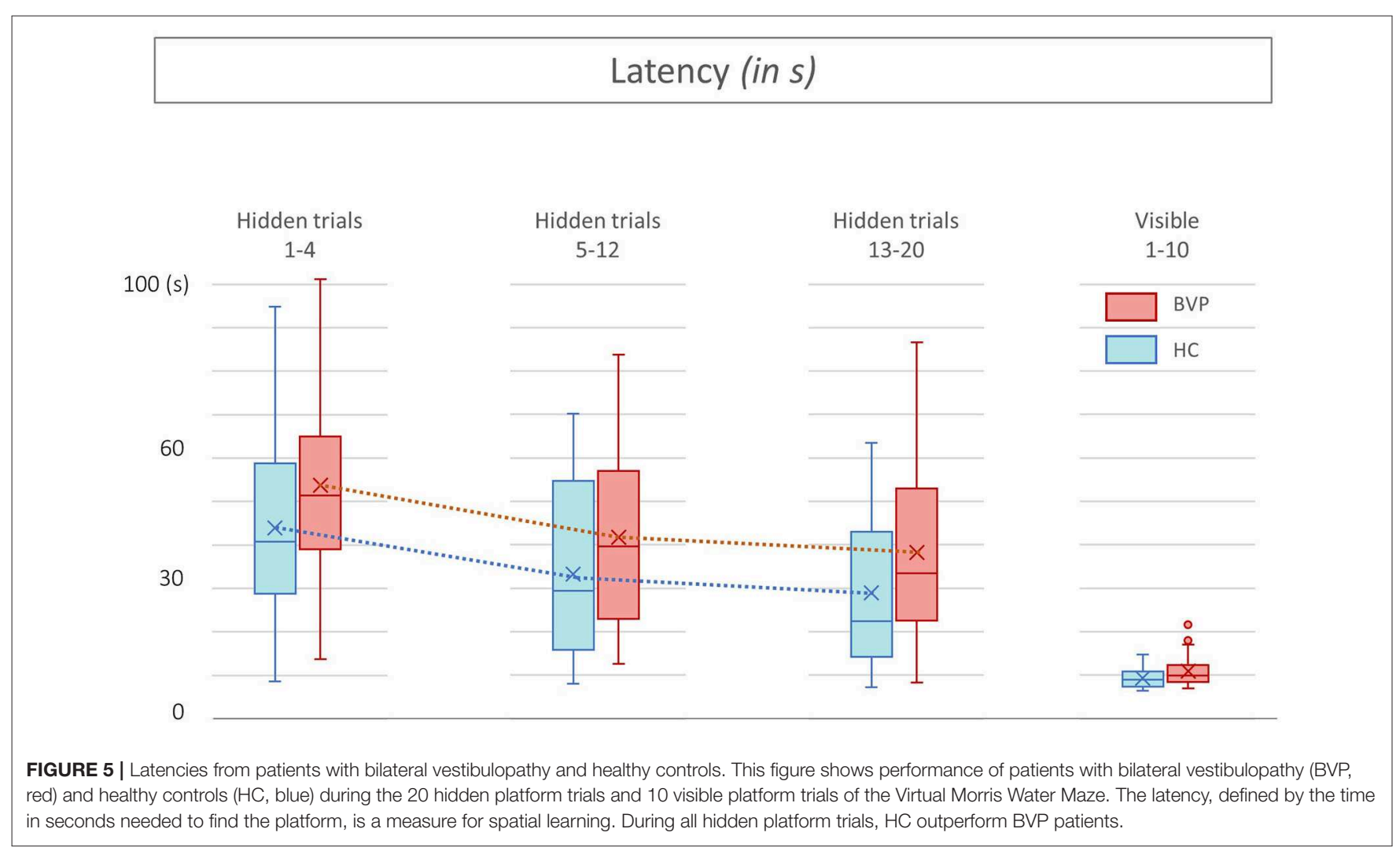




\section{Path length (in path length/pool diameter)}

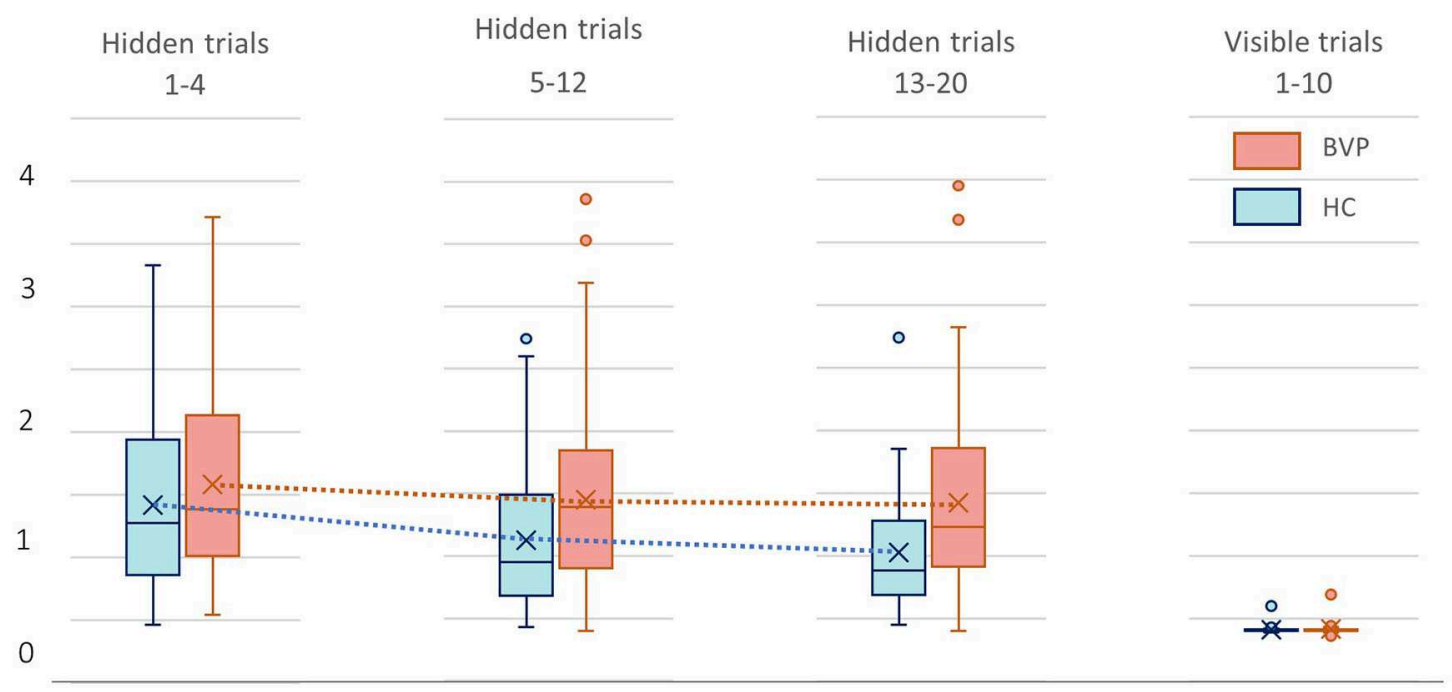

FIGURE 6 | Path length from patients with bilateral vestibulopathy and healthy controls. This figure shows performance of patients with bilateral vestibulopathy (BVP, red) and healthy controls ( $\mathrm{HC}$, blue) during the 20 hidden platform trials and 10 visible platform trials of the Virtual Morris Water Maze. The path length, defined by the relative distance to the pool diameter covered to reach the platform, is a measure for spatial learning. During all hidden platform trials, performance of BVP patients is worse than $\mathrm{HC}$.

healthy controls. Results of the visible trials are shown in Figures 5-7.

\section{DISCUSSION}

The present study was designed to evaluate whether BVP patients suffer from spatial cognitive deficits compared with healthy controls. Furthermore, the analyses in this study were especially set out with the aim of evaluating the importance of concomitant hearing loss of BVP patients regarding the suspected relationship between cognition and the vestibular system.

In one of the largest BVP patient group so far, this study found a worse performance on all outcome measures of the VMWT in BVP patients compared with healthy controls. However, this difference was never statistically significant between the BPV group and the healthy control group. In contrast to earlier studies, all statistical analyses of this study included correction for hearing (dys)function. Although no significant group difference was observed, it seemed that, on the other hand, hearing loss was found to be statistically significantly associated with worse spatial cognition. The worse the hearing of BVP patients, the worse the spatial learning indicated by longer latencies in the hidden trial of the VMWT. Likewise, in the probe trial, hearing loss resulted in less time spent in the platform quadrant, which suggests worse spatial memory retrieval.

\section{Vestibular Loss and Spatial Cognition}

Previous studies have not dealt with the hearing status of the enrolled BVP patients when drawing conclusions about the relationship between vestibular loss and cognitive decline (6). Given the observed statistically significant effect of hearing loss on spatial cognition, this study highlights the need to correct for hearing loss when evaluating cognition in vestibular patients. Furthermore, our findings raise intriguing questions regarding the assumed link between cognition and the vestibular system. According to our results, it could be questioned whether the spatial cognitive deficits of BVP patients might be solely attributed to their hearing loss and not to their vestibular loss. However, it is important to bear in mind that the control group in this study included subjects with normal age-appropriate hearing. Hence, some of the control subjects suffered from presbyacusis, but overall the prevalence of hearing loss in the control group is low. Therefore, results should be interpreted with caution and it cannot be concluded that hearing loss is the only factor resulting in the spatial cognitive deficits of BVP patients. Vestibular loss might play an additional role. Moreover, previous studies observing spatial deficits in BVP patients included patients with complete vestibular loss. In this study, patients with BVP, as defined by the Bárány criteria, were included. This implicates that also patients with partial vestibular loss were included, for example, preserved function on vHIT in the absence of caloric function (7). Future work 


\section{Heading error (in ${ }^{\circ}$ )}

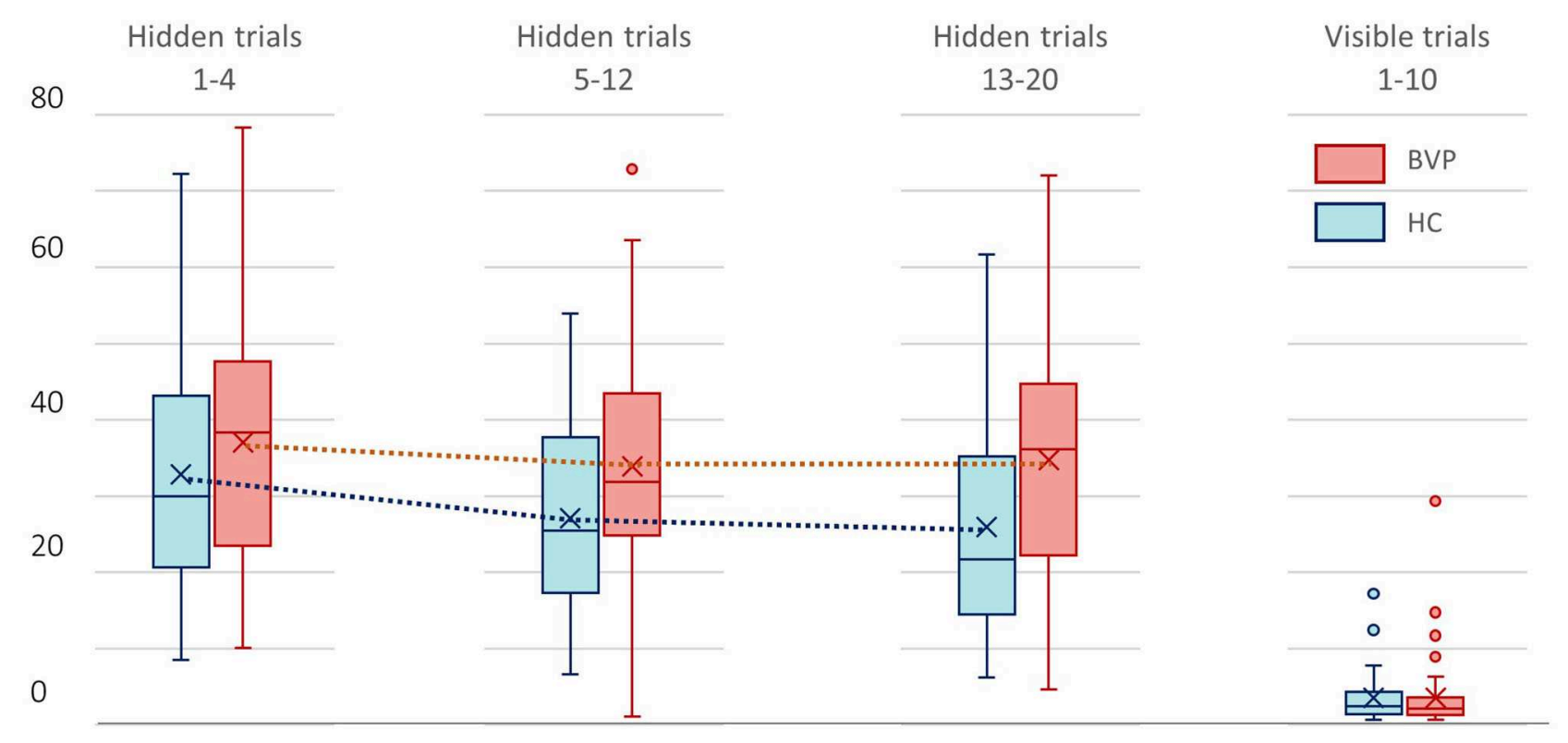

FIGURE 7 | Heading errors from patients with bilateral vestibulopathy and healthy controls. This figure shows the performance of patients with bilateral vestibulopathy (BVP, red) and healthy controls ( $\mathrm{HC}$, blue) during the 20 hidden platform trials and 10 visible platform trials of the Virtual Morris Water Maze. The higher the heading error, the worse the performance and thus spatial learning. In all hidden trials, the heading errors of BVP patients are higher than those of HC.

is required to further unravel the link between cognition, vestibular loss, and hearing loss. An interesting study protocol would be to compare spatial cognition between four groups: healthy controls, patients with hearing dysfunction and normal vestibular function, patients with normal hearing and vestibular dysfunction and finally, patients with both vestibular and hearing dysfunction.

Nonetheless, for all future studies investigating cognition in BVP patients, our results implicate that it is obligatory to take the hearing status of BVP patients into account.

\section{Hearing Loss, Spatial Cognition, and the Hippocampus}

In accordance with the present results, previous studies have demonstrated a link between spatial cognition and hearing loss. A recent meta-analysis showed a significant impairment of visuospatial abilities in patients with hearing loss across cross-sectional studies, using a wide variety of spatial cognitive tasks (26).

Two recent animal studies investigated spatial cognition using the Morris Water Maze $(36,37)$. Mice with presbyacusis were found to have worse spatial learning and spatial memory retrieval compared with mice with normal hearing (36). Likewise, mice with noise-induced hearing loss showed poorer performance during the Morris Water Maze (37). This was pointed out by longer latencies during the hidden platform trials and less time spent in the platform quadrant during a probe trial in mice with noise-induced hearing loss compared with mice with normal hearing. It is important to note that mice typically do not perform well in the Morris Water Maze and authors suggest that they might not use spatial strategy. Hence, caution should be taken to extrapolate these findings to humans (38).

The hippocampus is the area of the brain that has long been implicated in spatial memory. Animal and human studies have shown altered functioning and even atrophy of the hippocampus in subjects with vestibular loss [for review see (4)]. Interestingly, the poorer spatial performance of mice with hearing loss was also accompanied by a decrease of hippocampal neurogenesis (37). As the Morris Water Maze does not rely upon auditory function, authors hypothesize that the auditory input plays a maintenance role for hippocampal function and neurogenesis (37). However, it should be noted that exposing mice to noise trauma does not only result in hearing loss but might also induce peripheral vestibular damage $(39,40)$. This has not been taken into account in the study. Hence, it is possible that the decreased hippocampal neurogenesis observed in mice with noise-induced hearing loss is (partially) related to a loss of peripheral vestibular input. Vice versa, despite the extensive previous research, many questions remain about the neuroanatomical substrate underlying the association between 
the vestibular system, spatial cognition, and the hippocampus. Little research has been carried out to investigate if subjects with hearing loss have altered hippocampal function and volume. Hence, it could conceivably be hypothesized that hearing loss plays a role in the assumed neuroanatomical pathways between

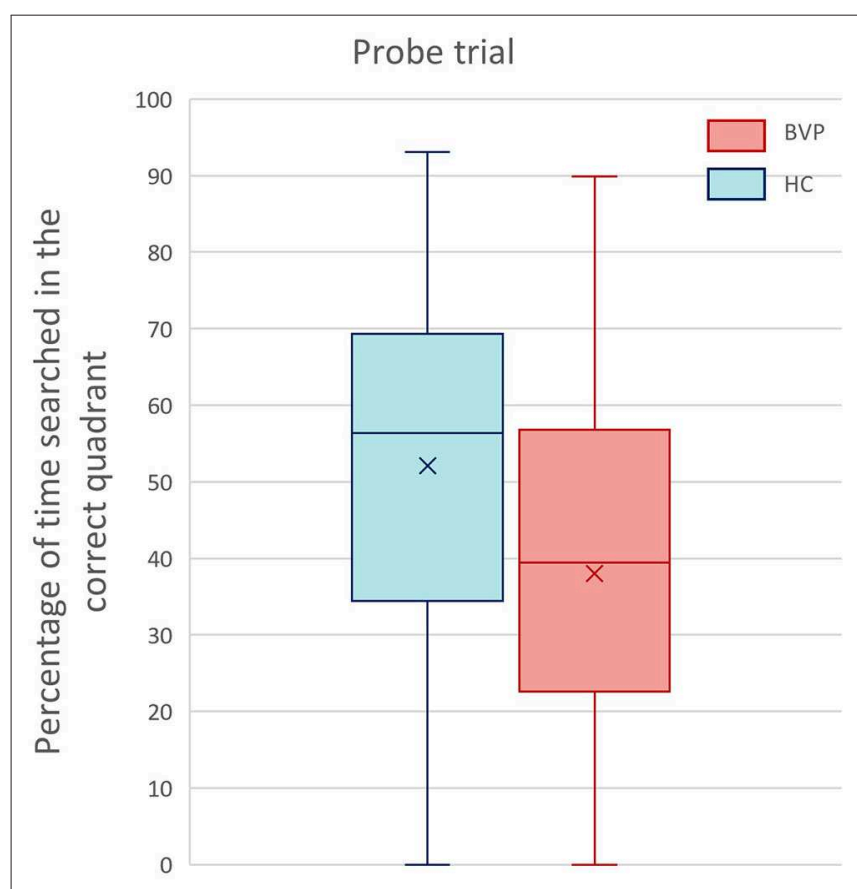

FIGURE 8 | Results of the probe trial of the Virtual Morris Water Task. This boxplot shows the performance of BVP patients (red) and healthy controls (blue) during the probe trial. Spatial memory retrieval was defined by the relative amount of search time (\%) spent in the correct quadrant of the pool. the peripheral vestibular input and the hippocampal and cortical areas involved in spatial cognition.

\section{The VMWT to Assess Spatial Cognition in BVP Patients}

In previous literature, the VMWT seemed to be one of the most used tools to assess spatial cognition in vestibular patients (2). In patients with complete loss of vestibular input after a bilateral vestibular neurectomy, distinct poorer performance was observed on the hidden and probe trials of the VMWT (7). In a more recent study of the same group, patients with severe but incomplete BVP showed more subtle spatial cognitive deficits (3). Likewise, in patients with a unilateral loss of vestibular input, only one of the outcome measures was impaired in patients with right unilateral vestibulopathy. In the patients with left unilateral vestibulopathy, none of the outcome measures differed significantly from controls (8). These results suggest that with increasing loss of peripheral vestibular input, the spatial cognition decreases. In our study, most BVP patients suffered from a deep but incomplete loss of vestibular function. It is possible, therefore, that the worse VMWT performance in our BVP patients did not yield statistically significance level.

Furthermore, the purely stationary set-up of the VMWT might underestimate the real-life spatial cognitive deficits of BVP patients as a result of loss of vestibular input. Previous research has established that, while navigating, an "inner neural map" is created, based on peripheral vestibular input. This neural representation of the external environment is computed in the hippocampus and entorhinal cortex and consists of several cooperative cell types: angular head velocity cells; head direction cells; place and grid cells (41). Rodent studies have demonstrated that vestibular input modulates the activity of the head direction cells and the place cells $(33,42)$. As the VMWT is static, the task does not rely on any vestibular input from real locomotion.

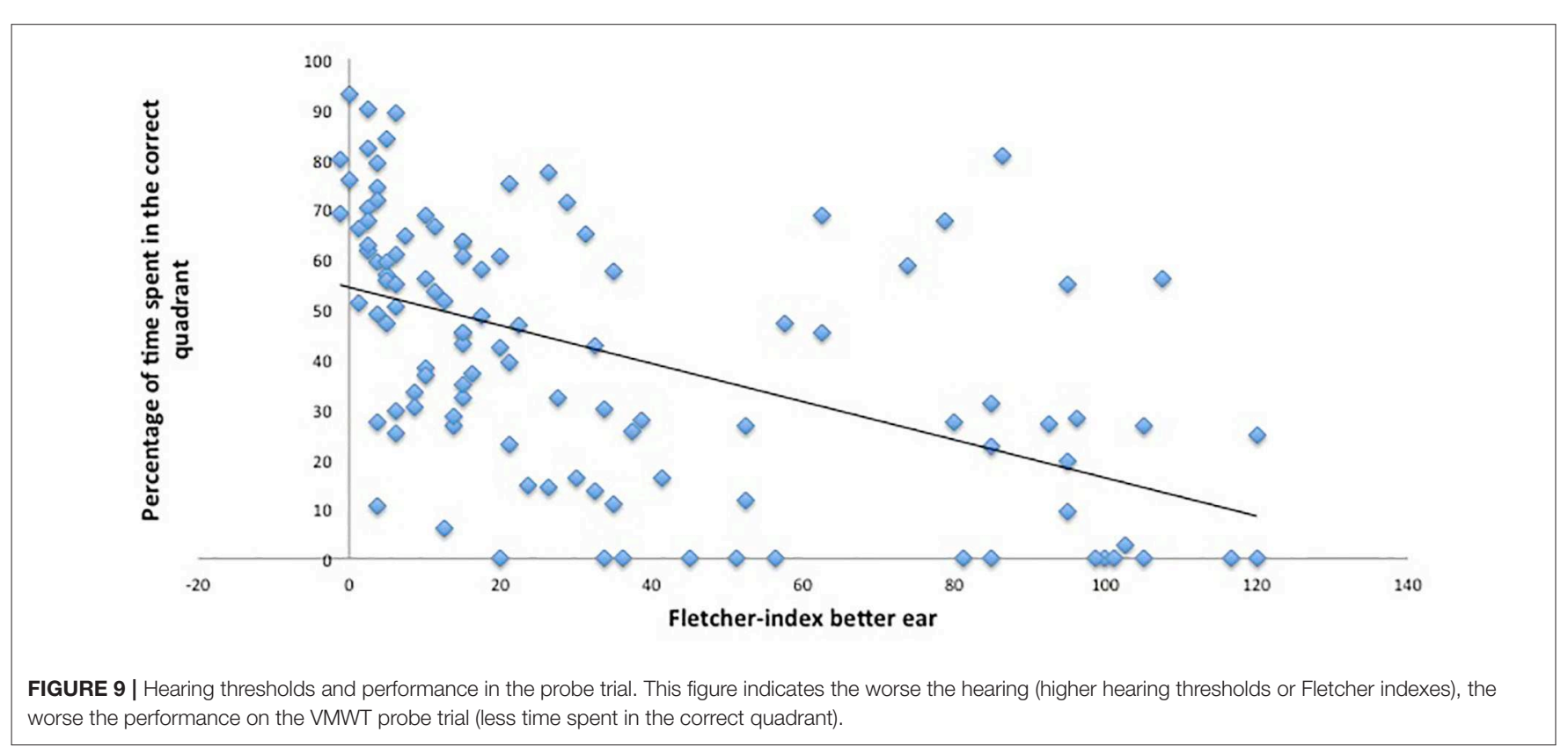


Hence, it is likely that real-life navigation tasks will be more sensitive to reveal spatial cognitive deficits in BVP patients.

Moreover, attentional deficits are demonstrated in BVP patients $(3,6,43)$. According to Kahneman's Capacity Model of Attention in which an individual has a limited total amount of cognitive resources available to divide among mental tasks, dual tasking might be more demanding in BVP patients because of the increased attentional need for keeping balance (34). As subjects stay seated during the VMWT, attentional resources can be fully directed toward the spatial memory task. This might be a second reason why the VMWT underestimates the real-life spatial cognitive deficits of BVP patients.

To sum up, the VMWT is a widely used method to assess spatial cognition in humans (9). Several studies have demonstrated spatial cognitive deficits in BVP patients, using the VMWT $(3,7,8)$. However, previous studies have not dealt with the concomitant hearing loss of BVP patients. As hearing loss is a risk factor for dementia, this might be an important forgotten factor. This is the first study investigating spatial cognition by use of the VMWT in a BVP group as large as 64 patients, and with correction for the hearing (dys)function in all analyses. All outcome measures of the VMWT were worse in BVP patients compared with healthy controls; however, these differences were not statistically significant. Contrarily, hearing loss was statistically significantly associated with worse spatial learning and spatial memory retrieval. Regarding our study protocol with healthy controls without severe hearing loss, it is not excluded that vestibular loss has an additional effect on spatial cognition. Nonetheless, our findings confirm the negative repercussion of hearing loss on spatial cognition (26), and highlight the need to correct for hearing loss when investigating cognition in a vestibular population group.

Both vestibular and hearing dysfunction are prevalent in the elderly $(32,44,45)$. Given the rising prevalence of dementia, and the current lack of therapy, future studies are needed to identify modifiable risk factors (16). Therefore, the link between cognitive decline and the hippocampus on the one hand and hearing loss and vestibular loss on the other hand needs to be further unraveled. To develop a full picture, a study protocol that would additionally include patients with normal vestibular function but different levels of sensorineural hearing loss would be interesting. Furthermore, considering the static and single task paradigm involved in the VMWT, real navigation tasks might give more insights in the potential spatial cognitive deficits related to the loss of vestibular input.

\section{Limitations}

The subjects in our HC group were not perfectly matched to BVP patients regarding age, education, and computer experience. As HC were on average younger, more educated, and more computer experienced, VMWT performance could be relatively overestimated in the HC group. Regarding the observed negative effect of hearing loss on spatial cognition (longer latencies and less time spent in the correct quadrant), it is important to bear in mind that the majority of patients with hearing loss were in the BVP group. Therefore, it is not excluded that vestibular loss plays an additional role in spatial cognition, which could not be observed in this study protocol using healthy controls with normal hearing. Second, there was a correlation with hearing loss and age. Although all models were corrected for age, it is not excluded that age might play an important role in the observed link between spatial cognition and hearing loss.

\section{CONCLUSION}

The present study assesses spatial cognitive performance in one of the largest BVP cohorts so far. The study was especially designed to determine the relative importance of hearing loss in spatial cognition of BVP patients, as this has been frequently overlooked. We found worse spatial cognitive performance on all outcome measures of BVP patients. However, these differences were not statistically significant between the BVP patients and healthy controls, when corrected for age, gender, education, level of computer use, and hearing loss. Interestingly, only hearing loss was found to be statistically significantly associated with worse spatial cognition. These findings highlight the need to correct for hearing loss in future studies investigating cognition in BVP patients. As the control group did not include subjects with severe hearing loss, an additional effect of vestibular loss on spatial cognitive performance cannot be excluded.

\section{ADDITIONAL COMMENTS}

These data have partially been presented on the 30th Bárány conference, Uppsala, June 2018.

\section{DATA AVAILABILITY STATEMENT}

The raw data supporting the conclusions of this article will be made available by the authors, without undue reservation.

\section{ETHICS STATEMENT}

The studies involving human participants were reviewed and approved by local ethics committee of the Antwerp University Hospital/University of Antwerp (protocol number 16/42/426). The patients/participants provided their written informed consent to participate in this study.

\section{AUTHOR CONTRIBUTIONS}

BD: data collection, statistical analyses, study concept, and writing manuscript. GM, RB, and PV: study concept and supervision. JM: data collection. EF: study concept and statistical analyses. VV: study concept, writing manuscript, and supervision. All authors contributed to the article and approved the submitted version.

\section{FUNDING}

The Antwerp University Hospital and Maastricht University Medical Center have received research and travel grants from MED-EL. The funders had no role in study design, data collection and analysis, decision to publish, or preparation of the manuscript. 


\section{REFERENCES}

1. Smith PF, Zheng Y. From ear to uncertainty: vestibular contributions to cognitive function. Front Integr Neurosci. (2013) 7:84. doi: 10.3389/fnint.2013.00084

2. Bigelow RT, Agrawal Y. Vestibular involvement in cognition: visuospatial ability, attention, executive function, and memory. J Vestib Res. (2015) 25:7389. doi: 10.3233/VES-150544

3. Kremmyda O, Hufner K, Flanagin VL, Hamilton DA, Linn J, Strupp M, et al. Beyond dizziness: virtual navigation, spatial anxiety and hippocampal volume in bilateral vestibulopathy. Front Hum Neurosci. (2016) 10:139. doi: 10.3389/fnhum.2016.00139

4. Smith PF. The vestibular system and cognition. Curr Opin Neurol. (2017) 30:84-9. doi: 10.1097/WCO.0000000000000403

5. Sachdev PS, Blacker D, Blazer DG, Ganguli M, Jeste DV, Paulsen JS, et al. Classifying neurocognitive disorders: the DSM-5 approach. Nat Rev Neurol. (2014) 10:634-42. doi: 10.1038/nrneurol.2014.181

6. Dobbels BPO, Boon B, Mertens G, Van De Heyning P, Van Rompaey V. Impact of bilateral vestibulopathy on spatial and nonspatial cognition: a systematic review. Ear Hear. (2018) 40:757-65. doi: 10.1097/AUD.0000000000000679

7. Brandt T, Schautzer F, Hamilton DA, Bruning R, Markowitsch HJ, Kalla $\mathrm{R}$, et al. Vestibular loss causes hippocampal atrophy and impaired spatial memory in humans. Brain. (2005) 128:2732-41. doi: 10.1093/brain/awh617

8. Hufner K, Hamilton DA, Kalla R, Stephan T, Glasauer S, Ma J, et al. Spatial memory and hippocampal volume in humans with unilateral vestibular deafferentation. Hippocampus. (2007) 17:471-85. doi: 10.1002/hipo.20283

9. Hamilton DA, Driscoll I, Sutherland RJ. Human place learning in a virtual Morris water task: some important constraints on the flexibility of place navigation. Behav Brain Res. (2002) 129:159-70. doi: 10.1016/S0166-4328(01)00343-6

10. Lucieer F, Duijn S, Van Rompaey V, Perez Fornos A, Guinand N, Guyot JP, et al. Full spectrum of reported symptoms of bilateral vestibulopathy needs further investigation-a systematic review. Front Neurol. (2018) 9:352. doi: 10.3389/fneur.2018.00352

11. Fawzy M, Khater A. Bilateral vestibulopathy treatment: update and future directions. Egypt J Otolaryngol. (2016) 32:83-92. doi: 10.4103/1012-5574.181082

12. Hain TC, Cherchi M, Yacovino DA. Bilateral vestibular loss. Semin Neurol. (2013) 33:195-203. doi: 10.1055/s-0033-1354597

13. Lee YM, Jang C, Bak IH, Yoon JS. Effects of computer-assisted cognitive rehabilitation training on the cognition and static balance of the elderly. J Phys Ther Sci. (2013) 25:1475-7. doi: 10.1589/jpts.25.1475

14. Smith-Ray RL, Irmiter C, Boulter K. Cognitive training among cognitively impaired older adults: a feasibility study assessing the potential improvement in balance. Front Public Health. (2016) 4:219. doi: 10.3389/fpubh.2016. 00219

15. Ellis AW, Schone CG, Vibert D, Caversaccio MD, Mast FW. Cognitive rehabilitation in bilateral vestibular patients: a computational perspective. Front Neurol. (2018) 9:286. doi: 10.3389/fneur.2018.00286

16. Alzheimer's A. 2016 Alzheimer's disease facts and figures. Alzheimers Dement. (2016) 12:459-509. doi: 10.1016/j.jalz.2016.03.001

17. Halliday G. Pathology and hippocampal atrophy in Alzheimer's disease. Lancet Neurol. (2017) 16:862-4. doi: 10.1016/S1474-4422(17)30343-5

18. Birdane L, Incesulu A, Gurbuz MK, Ozbabalik D. Sacculocolic reflex in patients with dementia: is it possible to use it for early diagnosis? Neurol Sci. (2012) 33:17-21. doi: 10.1007/s10072-011-0595-3

19. Nakamagoe K, Fujimiya S, Koganezawa T, Kadono K, Shimizu K, Fujizuka N, et al. Vestibular function impairment in Alzheimer's disease. J Alzheimers Dis. (2015) 47:185-96. doi: 10.3233/JAD-142646

20. Harun A, Oh ES, Bigelow RT, Studenski S, Agrawal Y. Vestibular impairment in dementia. Otol Neurotol. (2016) 37:1137-42. doi: 10.1097/MAO.0000000000001157

21. Livingston G, Sommerlad A, Orgeta V, Costafreda SG, Huntley J, Ames D, et al. Dementia prevention, intervention, and care. Lancet. (2017) 390:2673734. doi: 10.1016/S0140-6736(17)31363-6

22. Zingler VC, Cnyrim C, Jahn K, Weintz E, Fernbacher J, Frenzel C, et al. Causative factors and epidemiology of bilateral vestibulopathy in 255 patients. Ann Neurol. (2007) 61:524-32. doi: 10.1002/ana.21105
23. Lucieer F, Vonk P, Guinand N, Stokroos R, Kingma H, Van De Berg R. Bilateral vestibular hypofunction: insights in etiologies, clinical subtypes, and diagnostics. Front Neurol. (2016) 7:26. doi: 10.3389/fneur.2016. 00026

24. Claes AJ, Van De Heyning P, Gilles A, Hofkens-Van Den Brandt A, Van Rompaey V, Mertens G. Impaired cognitive functioning in cochlear implant recipients over the age of 55 years: a cross-sectional study using the repeatable battery for the assessment of neuropsychological status for hearing-impaired individuals (RBANS-H). Front Neurosci. (2018) 12:580. doi: $10.3389 /$ fnins.2018.00580

25. Claes AJ, Van De Heyning P, Gilles A, Van Rompaey V, Mertens G. Cognitive performance of severely hearing-impaired older adults before and after cochlear implantation: preliminary results of a prospective, longitudinal cohort study using the RBANS-H. Otol Neurotol. (2018). doi: 10.1097/MAO.0000000000001936

26. Loughrey DG, Kelly ME, Kelley GA, Brennan S, Lawlor BA. Association of age-related hearing loss with cognitive function, cognitive impairment, and dementia: a systematic review and meta-analysis. JAMA Otolaryngol Head Neck Surg. (2018) 144:115-26. doi: 10.1001/jamaoto. 2017.2513

27. Dobbels B, Mertens G, Gilles A, Claes A, Moyaert J, Van De Berg R, et al. Cognitive function in acquired bilateral vestibulopathy: a cross-sectional study on cognition, hearing, and vestibular loss. Front Neurosci. (2019) 13:340. doi: 10.3389 /fnins. 2019.00340

28. Strupp M, Kim JS, Murofushi T, Straumann D, Jen JC, Rosengren SM, et al. Bilateral vestibulopathy: diagnostic criteria consensus document of the Classification Committee of the Barany Society. J Vestib Res. (2017) 27:177-89. doi: 10.3233/VES-170619

29. Van Der Stappen A, Wuyts FL, Van De Heyning PH. Computerized electronystagmography: normative data revisited. Acta Otolaryngol. (2000) 120:724-30. doi: 10.1080/000164800750000243

30. Macdougall HG, Mcgarvie LA, Halmagyi GM, Curthoys IS, Weber KP. The video Head Impulse Test (vHIT) detects vertical semicircular canal dysfunction. PLoS ONE. (2013) 8:e61488. doi: 10.1371/journal.pone.0061488

31. Morris R. Spatial localization does not require the presence of local cues. Learn Motiv. (1981) 12:239-60. doi: 10.1016/0023-9690(81) 90020-5

32. Lin FR, Thorpe R, Gordon-Salant S, Ferrucci L. Hearing loss prevalence and risk factors among older adults in the United States. J Gerontol A Biol Sci Med Sci. (2011) 66:582-90. doi: 10.1093/gerona/glr002

33. Yoder RM, Taube JS. The vestibular contribution to the head direction signal and navigation. Front Integr Neurosci. (2014) 8:32. doi: $10.3389 /$ fnint.2014.00032

34. Bigelow RT, Semenov YR, Trevino C, Ferrucci L, Resnick SM, Simonsick EM, et al. Association between visuospatial ability and vestibular function in the Baltimore longitudinal study of aging. J Am Geriatr Soc. (2015) 63:1837-44. doi: 10.1111/jgs.13609

35. De Belder J, Matthysen S, Claes AJ, Mertens G, Van De Heyning P, Van Rompaey V. Does otovestibular loss in the autosomal dominant disorder DFNA9 have an impact of on cognition? A systematic review. Front Neurosci. (2017) 11:735. doi: 10.3389/fnins.2017.00735

36. Dong $\mathrm{Y}$, Guo CR, Chen $\mathrm{D}$, Chen SM, Peng $\mathrm{Y}$, Song $\mathrm{H}$, et al. Association between agerelated hearing loss and cognitive decline in C57BL/6J mice. Mol Med Rep. (2018) 18:1726-32. doi: 10.3892/mmr. 2018.9118

37. Liu L, Xuan C, Shen P, He T, Chang Y, Shi L, et al. Hippocampal mechanisms underlying impairment in spatial learning long after establishment of noiseinduced hearing loss in CBA mice. Front Syst Neurosci. (2018) 12:35. doi: 10.3389/fnsys.2018.00035

38. Whishaw IQ, Tomie J. Of mice and mazes: similarities between mice and rats on dry land but not water mazes. Physiol Behav. (1996) 60:1191-7. doi: 10.1016/S0031-9384(96)00176-X

39. Stewart C, Yu Y, Huang J, Maklad A, Tang X, Allison J, et al. Effects of high intensity noise on the vestibular system in rats. Hear Res. (2016) 335:118-27. doi: 10.1016/j.heares.2016.03.002

40. Stewart CE, Kanicki AC, Altschuler RA, King WM. Vestibular short-latency evoked potential abolished by low-frequency noise exposure in rats. $J$ Neurophysiol. (2018) 119:662-7. doi: 10.1152/jn.00668.2017 
41. Brandt T, Zwergal A, Glasauer S. 3-D spatial memory and navigation: functions and disorders. Curr Opin Neurol. (2017) 30:90-7. doi: 10.1097/WCO.0000000000000415

42. Stackman RW, Clark AS, Taube JS. Hippocampal spatial representations require vestibular input. Hippocampus. (2002) 12:291-303. doi: 10.1002/hipo.1112

43. Popp P, Wulff M, Finke K, Ruhl M, Brandt T, Dieterich M. Cognitive deficits in patients with a chronic vestibular failure. J Neurol. (2017) 264:554-63. doi: 10.1007/s00415-0168386-7

44. Agrawal Y, Carey JP, Della Santina CC, Schubert MC, Minor LB, Disorders of balance and vestibular function in US adults: data from the National Health and Nutrition Examination Survey, 2001-2004. Arch Intern Med. (2009) 169:938-44. doi: 10.1001/archinternmed. 2009.66
45. Agrawal Y, Van de Berg R, Wuyts F, Walther L, Magnusson M, Oh E, et al. Presbyvestibulopathy: diagnostic criteria. J Vestibul Res. (2018) 29:161-70. doi: 10.3233/VES-190672

Conflict of Interest: The authors declare that the research was conducted in the absence of any commercial or financial relationships that could be construed as a potential conflict of interest.

Copyright (C) 2020 Dobbels, Mertens, Gilles, Moyaert, van de Berg, Fransen, Van de Heyning and Van Rompaey. This is an open-access article distributed under the terms of the Creative Commons Attribution License (CC BY). The use, distribution or reproduction in other forums is permitted, provided the original author(s) and the copyright owner(s) are credited and that the original publication in this journal is cited, in accordance with accepted academic practice. No use, distribution or reproduction is permitted which does not comply with these terms. 